\title{
Establishment of a novel orthotopic model of breast cancer metastasis to the lung
}

\author{
WENLI GUO ${ }^{1}$, SHUPING ZHANG ${ }^{2}$ and SIJIN LIU ${ }^{1}$
${ }^{1}$ State Key Laboratory of Environmental Chemistry and Ecotoxicology, Research Center for Eco-Environmental Sciences, Chinese Academy of Sciences, Beijing 100085, P.R. China; ${ }^{2}$ Institute for Medical Engineering and Science, \\ Massachusetts Institute of Technology, Cambridge, MA 02139, USA
}

Received February 15, 2015; Accepted March 23, 2015

DOI: $10.3892 /$ or.2015.3927

\begin{abstract}
Breast cancer is the second leading cause of cancer-related death among women, and distant metastasis is responsible for the death of $\sim 90 \%$ of these patients. However, despite recent advances, the underlying mechanisms responsible for breast cancer metastasis remain elusive. A great impediment to this is the lack of appropriate orthotopic models of breast cancer metastasis to distant organs. In the present study, we established a novel orthotopic model of breast cancer metastasis to the lungs in mice, where metastatic sublines of 4T1 cells revealed enhanced metastatic propensity to the lungs. All mice $(100 \%)$ developed lung metastasis upon orthotopic implantation of a metastatic subline of 4T1 cells, in contrast to $10 \%$ of mice with lung metastasis for a subline derived from primary tumors and $60 \%$ of mice with lung metastasis for parental 4T1 cells. At the molecular level, significant epithelial-mesenchymal transition was characterized in these metastatic sublines, which is likely at least partially, responsible for the enhanced metastasis. These established cell lines provide a novel platform to study the relevant molecular basis of metastasis and metastasis-specific therapeutics.
\end{abstract}

\section{Introduction}

Cancer remains one of the most common causes of death worldwide, and breast cancer is the most commonly diagnosed cancer type, and a leading cause of cancer-related deaths in women, particularly in western countries $(1,2)$. Despite numerous inspiring advances in the past years, the underlying mechanisms of breast cancer initiation and progression remain elusive. Moreover, once cancer cells form secondary tumors in

Correspondence to: Professor Sijin Liu, State Key Laboratory of Environmental Chemistry and Ecotoxicology, Research Center for Eco-Environmental Sciences, Chinese Academy of Sciences, Beijing 100085, P.R. China

E-mail: sjliu@rcees.ac.cn

Key words: breast cancer, metastasis, orthotopic model, lung, epithelial-mesenchymal transition other organs, the disease essentially enters an incurable phase. Metastatic tumors are currently treated as a systemic disease with conventional therapeutics, such as chemotherapy and/ or radiation. Although these strategies ameliorate metastatic progression and relevant implications, it is impossible to stop or reverse the progression of metastatic disease. To this end, tremendous efforts have been invested in search of therapeutics for breast cancer metastasis, and some progress has been achieved to date. For example, zoledronic acid (Zometa ${ }^{\circledR}$ ) has been promisingly used as an adjuvant therapy to treat breast cancer with metastasis, in combination with chemotherapy, and it has significantly improved patient prognosis $(3,4)$. However, no metastasis-specific therapeutics exist. Therefore, further research on breast cancer metastasis should be conducted.

To study the metastatic process requires detailed insights into the cellular and molecular traits for both primary and metastatic tumors, as their differences determine the fundamental metastatic propensity. However, it is difficult to perform such studies with human primary breast tumors and corresponding metastatic tumors concomitantly, since these paired samples are mostly unavailable for laboratory investigation. Thus, it is desirable to establish mouse models of breast cancer metastasis. To date, several breast cancer cell lines have been established with enhanced inclination to metastasize to distant organs. For example, a derivative subline (termed 4175) of human MDA-MB-231 cells was established with strong inclination for lung metastasis in BALB/c nude mice $(5,6)$. It should be noted that research results from mice may not be able to reflect corresponding changes in humans, and the mechanisms responsible for the metastases of human breast cancer cells to mouse organs should be distinct from those to human organs. Thus, it would be more informative for mechanistic studies to use the same species of breast cancer cells and recipients. To this end, mouse breast cancer cells would be the perfect fit for this purpose. By contrast, there are a limited number of mouse breast cancer cell lines available for experimental studies. Among these, 4T1 cells have been widely used for the study of breast cancer development and progression, and these cells pose a propensity to metastasize to distant organs $(7,8)$. When inoculated orthotopically in mammary fat pads (MFPs), 4T1 cells spontaneously metastasize to several organs including the lung, liver, brain and bone $(7,9,10)$. However, it takes a long time (e.g. 5-6 weeks) for the parental 4T1 cells to 
form metastatic tumors in distant organs in mice, when growth regression of primary tumors has occurred (3-4 weeks) with extensive necrosis and infiltration of leukocytes (8). Moreover, the occurrence of metastases is limited in mice after introduction of parental $4 \mathrm{~T} 1$ cells at MFPs $(7,11)$. Therefore, sibling cell lines of 4T1 cells with enhanced metastatic properties are desirable for the study of the molecular basis of tumor metastasis. In the present study, we aimed to isolate and establish sublines of 4T1 cells that exhibit enhanced propensity to metastasize. We purified metastatic tumor cells from lungs, and eventually established sublines with enhanced capability to metastasize to the lungs.

\section{Materials and methods}

Cell culture. Mouse breast cancer 4T1 cells were provided as a gift from the Caliper Life Sciences, Inc. The genetically engineered cells are stably tagged with the luciferase gene. Cells were cultured in RPMI-1640 (Gibco) media, supplemented with $10 \%$ fetal bovine serum (FBS) and $100 \mathrm{U} / \mathrm{ml}$ penicillin-streptomycin (both from HyClone) at $37^{\circ} \mathrm{C}$ under $5 \% \mathrm{CO}_{2}$.

Mouse experiments. All mouse experiments were approved by the Animal Ethics Committee of the Research Center for Eco-Environmental Sciences, Chinese Academy of Sciences. Mice with BALB/c genetic background were purchased from the Vital River Laboratories (Beijing, China). Mice were housed under specific pathogen-free (SPF) environment, and surgeries were performed under sterile conditions. For tumor cell inoculation, we followed a previously described method $(12,13)$. Briefly, $2.0 \times 10^{3}$ cells were implanted into the fourth mammary fat pads (MFPs) of the mice. Thereafter, the mice were monitored for bioluminescent imaging of luciferase activity every week using the Spectrum Imaging System and Living Image software (Caliper Life Sciences, Inc.). Prior to imaging, the mice were intraperitoneally administered $200 \mu \mathrm{l}$ luciferin (at $15 \mathrm{mg} / \mathrm{ml}$; PerkinElmer). Afterwards, the mice were imaged after anesthetization with isoflurane inhalation. Immediately after the mice were sacrificed, various organs were imaged.

Western blot analysis. Cells were collected and lysed in RIPA lysis buffer containing protease inhibitor cocktail (Roche). The concentration of total proteins was determined using the Lowry method. Equal amounts of protein lysates for each sample were subjected to SDS-PAGE. Thereafter, western blot analyses were performed following a standard protocol, as previously described $(12,13)$. Antibodies used here were against GAPDH (1:1,000; Santa Cruz), anti-Snail1 (1:500) and anti-occludin (1:500) (both from Bioss, Inc., China). GAPDH was used as a loading control for normalization. Chemiluminescent detection was performed by the ChemiDoc ${ }^{\mathrm{TM}} \mathrm{XRS}+$ System with Image $\mathrm{Lab}^{\mathrm{TM}}$ Software (Bio-Rad, Inc.). The intensity of the protein bands in the blots was determined with ImageJ software (NIH, Bethesda, MD, USA).

Cloning of tumor cells from primary tumors and metastatic tumors in the lungs. Firstly, primary tumors were gently removed and maintained in phosphate-buffered saline (PBS).
Thereafter, metastatic tumors were carefully dissected from the surrounding connective tissues in the lungs. We prepared a single-cell suspension of the tumor cells following a standard protocol, as previously described (14). Tumor cells were then cultured as their parental 4T1 cells.

Genomic DNA extraction and PCR analysis. To characterize the identity of the 4T1 sublines, genomic DNA was extracted from the sublines on the basis of the manufacturer's protocol (Qiagen). Then, as previously described (12), PCR analysis was carried out under the conditions as follows; $94^{\circ} \mathrm{C}$ for $5 \mathrm{~min}$; followed by $94^{\circ} \mathrm{C}$ for $30 \mathrm{sec}, 55^{\circ} \mathrm{C}$ for $35 \mathrm{sec}$ and $72^{\circ} \mathrm{C}$ for $35 \mathrm{sec}$ for 40 cycles; finally, an extension at $72^{\circ} \mathrm{C}$ for $5 \mathrm{~min}$. Thereafter, the PCR products were subjected to electrophoresis on a $1.5 \%$ agarose gel, followed by staining with ethidium bromide. Primers for PCR reactions were: puromycin F, 5'-ATGACCGAGTACAAGCCCAC-3' and R, 5'-GAGGCCT TCCATCTGTTGCT-3'; luciferase F, 5'-CGAGGCTACAAA CGCTCTCA-3' and R, 5'-CGAAGATGTTGGGGTGTTGC-3'.

Cell imaging for luciferase activity. Cells were collected and re-suspended in pre-warmed PBS. An equal number of cells in $100 \mu \mathrm{l}$ were re-seeded into 96 -well plates, followed by the addition of $100 \mu \mathrm{l}$ of luciferin $(300 \mu \mathrm{g} / \mathrm{ml})$. The plates were incubated at $37^{\circ} \mathrm{C}$ for $2 \mathrm{~min}$ before imaging with the Spectrum Imaging System.

Histological analysis. Hematoxylin and eosin (H\&E) staining was carried out following the standard protocol. Briefly, lung specimens were first fixed in 4\% PBS-buffered formaldehyde, and then embedded in paraffin. Sections were finally stained with $\mathrm{H} \& \mathrm{E}$ solutions.

Invasion and migration assays. The capability of tumor cell invasion and migration was determined with the transwell method, as previously described $(13,15)$. Briefly, $100 \mu \mathrm{l}$ of diluted matrigel (at $200 \mu \mathrm{g} / \mathrm{ml}$ ) was loaded onto the upper chambers of transwells, followed by incubation at $37^{\circ} \mathrm{C}$ for $3 \mathrm{~h}$. Afterwards, $5.0 \times 10^{4}$ cells were seeded onto the upper chambers in $100 \mu \mathrm{l}$ of serum-free medium. Thereafter, $600 \mu \mathrm{l}$ of medium with $10 \%$ FBS was added into the bottom wells. After the cells were cultured for $5 \mathrm{~h}$, the filters were removed from the upper chambers, and then fixed with $4 \%$ formaldehyde. The filters were stained with $5 \mu \mathrm{g} / \mathrm{ml}$ of 4,6-diamidino-2-phenylindole (DAPI) solution. Cell nuclei were visualized and counted under a fluorescence microscope.

Data analysis. Experimental data were analyzed with the SPSS Statistics 17.0 software package. Independent t-test was used to determine the difference between two groups. Data are shown as mean \pm standard error. Statistical significance was determined at $\mathrm{P}<0.05$.

\section{Results}

Purification of metastatic cells from metastatic tumors in the lungs. In an effort to obtain 4T1 cells with enhanced capability to specifically metastasize to the lungs, we endeavored to isolate metastatic tumors using a mouse model of breast cancer metastasis, as previously described by our research group (13). 


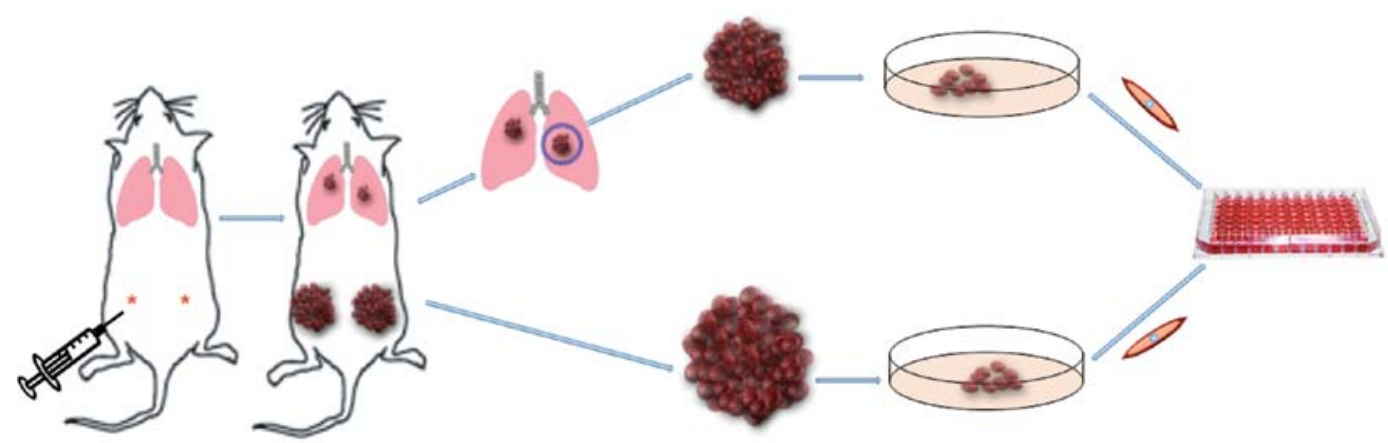

Figure 1. Scheme of the cloning of the sublines from the metastatic tumors in the lungs and from the primary tumors.

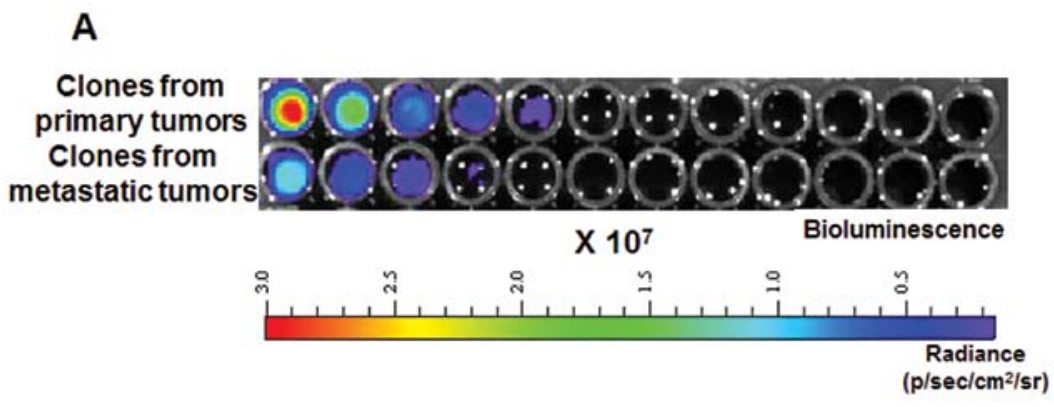

B

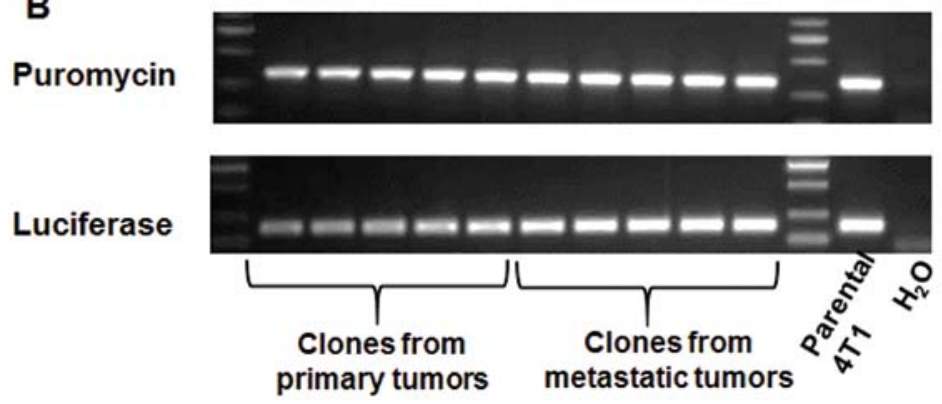

Figure 2. Identification of clones derived from metastatic tumors in lungs and from primary tumors. (A) Bioluminescent images showing luciferase activity in clones from metastatic tumors in lungs and from primary tumors. (B) PCR characterization of two marker genes, puromycin and luciferase, which were stably integrated into the genome of the parental 4T1 cells.

As shown in Fig. 1, a scheme was depicted for the purification and screening of metastatic cells from the metastatic tumors in the lungs in the present study. Six weeks post 4T1 cell inoculation in the bilateral MFPs, primary tumors reached $\sim 1 \mathrm{~cm}$ in diameter. When the mice were sacrificed, the primary tumors were dissociated and saved in PBS for later preparation of a single-cell suspension. Meanwhile, the lungs with marked tumors were also dissected, and then the tumors were carefully removed from the surrounding connective tissues. The generation of a single-cell suspension from the primary and metastatic tumors was performed with a standard protocol, as previously described (14). Cells were seeded in 96-well plates, and wells with a single cell/well were selected for subsequent subcloning and screening.

Subcloning of tumor cells with the capability of survival for an in vitro culture. The regimen for cell subcloning was similar to a previously described method (16). After colony formation for $\sim 2$ weeks, the 96 -well plates were examined for luciferase activity using the Spectrum Imaging System. As shown in Fig. 2A, the representative images showed a few clones with bright bioluminescence from the primary tumors and metastatic tumors. Afterwards, the identity of these sublines of 4T1 cells was assessed through PCR using two markers, namely puromycin and luciferase. Since these two markers were located in the original plasmid that was stably transfected into the 4T1 cells, they were detected only in the 4T1-derived cells. As shown in Fig. 2B, these cells were positive for these two markers, suggesting that they were sublines derived from the $4 \mathrm{~T} 1$ cells. These sublines were accordingly named as 4T1-P1, 4T1-P2, 4T1-P3, 4T1-P4 and 4T1-P5, representing sublines from the primary tumors, and 4T1-M1, 4T1-M2, 4T1-M3, 4T1-M4 and 4T1-M5, denoting sublines from the metastatic tumors.

Characterization of the sublines of the $4 T 1$ cells. Next, we characterized the metastatic propensity of these subclones. The migration/invasion capabilities were assessed with the 


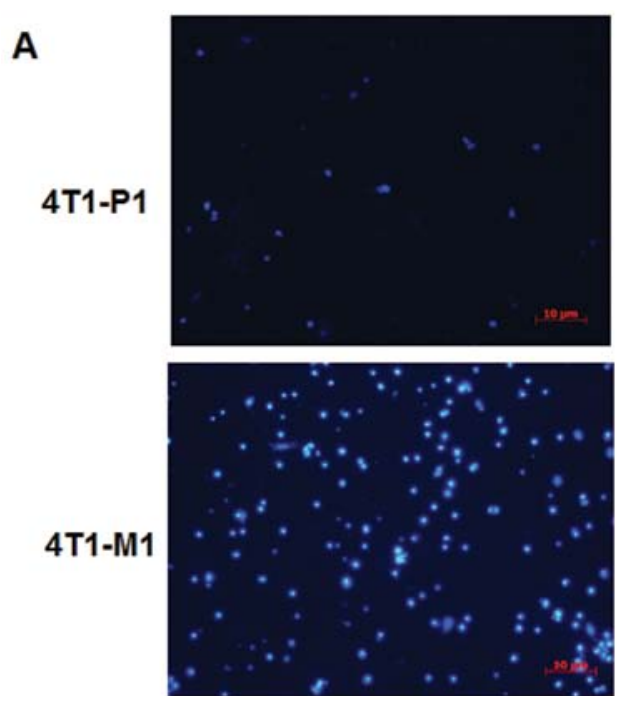

\section{B}

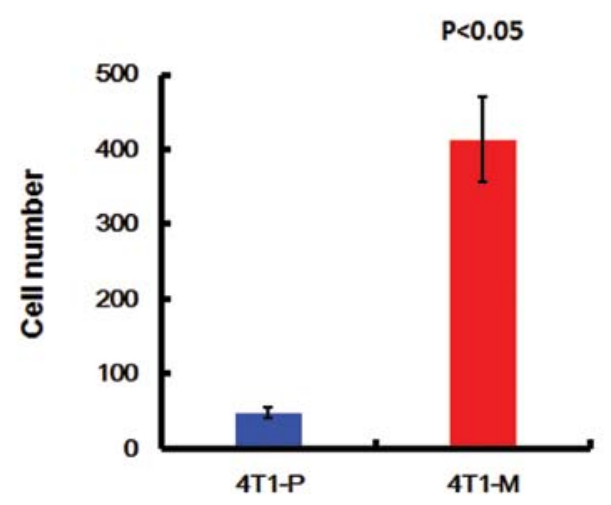

Figure 3. Evaluation of 4T1-P and 4T1-M cell migration/invasion. The capability of cell migration/invasion was assessed through the transwell method. (A) Representative images show transmigrated cells with DAPI staining. Original magnification, x100. (B) Quantitative data showing numbers of transmigrated cells. There were 3 replicates for each type of cells. Cell nuclei were counted in each field for 3 randomly selected fields in every filter, and an average was calculated for each sample $(n=3 \times 3)$.

A

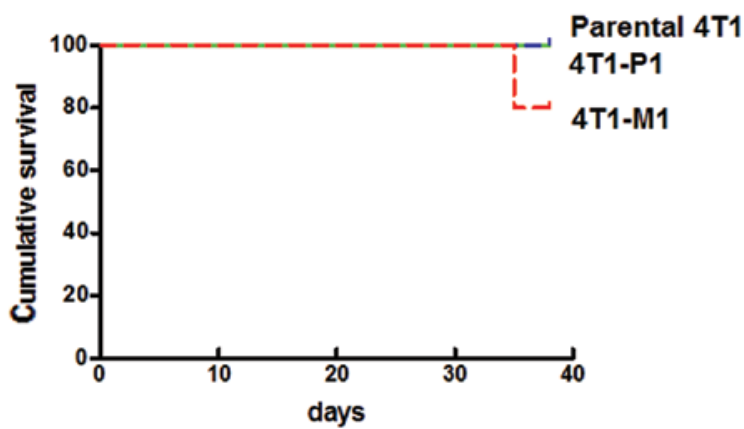

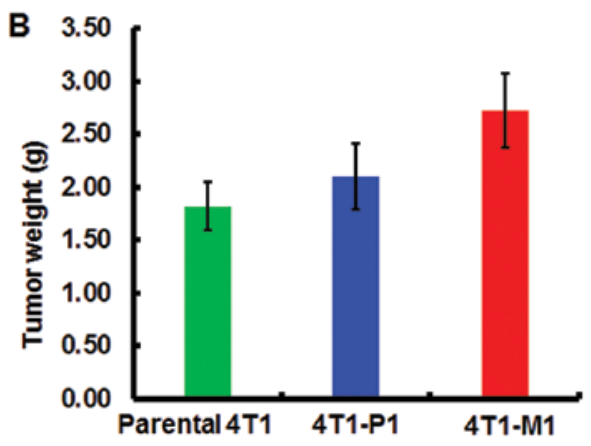

Figure 4. Tumor growth in mice inoculated with 4T1-P1 or 4T1-M1 cells. (A) The Kaplan-Meier survival curves of mice after inoculation of 4T1-P1 and 4T1-M1 cells. Survival of mice was monitored for 38 days $(n=10)$. (B) Final tumor weight of mice upon inoculation of parental 4T1, 4T1-P1 or 4T1-M1 cells (n=10).

transwell method, as previously described $(12,13)$. As reflected by the transwell results, the number of transmigrated cells was substantially increased for sublines from the metastatic tumors, compared to sublines from the primary tumors (Fig. 3A). Fig. 3A shows the representative images of the transmigrated cells with DAPI staining for the 4T1-P1, in comparison to the 4T1-M1 cells. The quantitative data indicated nearly a 9-fold difference between these two groups (Fig. 3B, P<0.05). These data demonstrated that the in vitro mobility and invasion capabilities were significantly enhanced in sublines from the metastatic tumors, compared to those from the primary tumors.

Elevated propensity of metastatic sublines of $4 T 1$ cells to the lungs. To validate the in vitro results, we thereafter selected two representative sublines, i.e. 4T1-M1 and 4T1-P1, for the in vivo study. We chose these two sublines since both displayed the strongest phenotypes in their own groups. In other words, 4T1-M1 exhibited the greatest capability to migrate and invade, in contrast, 4T1-P1 showed the weakest capability of migration and invasion through the transwell assay. We orthotopically inoculated 4T1-M1, 4T1-P1 and parental 4T1 cells into MFPs of the mice. During the 38-day experiment, the mice from all 3 groups developed primary tumors with $100 \%$ tumor occurrence. Two mice in the 4T1-M1 group died at the late stage of the experiment (Fig. 4A). Therefore, the survival rate was $80 \%$, in contrast to a $100 \%$ survival rate in the other two groups (Fig. 4A), suggesting reduced prognosis for mice upon introduction of the 4T1-M1 cells. The final tumor weight was increased in the mice inoculated with the 4T1-M1 cells, compared to the other two groups (Fig. 4B), despite of no statistical significance. Furthermore, we examined the occurrence of metastatic tumors in the lungs through bioluminescent imaging and histological examination. As shown in Fig. 5A, all mice harbored metastatic tumors in the lungs in the group of 4T1-M1, namely $100 \%$ lung metastasis for 4T1-M1 cells. In contrast, only $10 \%$ of the mice developed lung metastasis upon inoculation of the 4T1-P1 cells. Meanwhile, the inoculation of parental 4T1 cells caused $60 \%$ of lung metastasis, higher than that of parental cells, but lower than that of 4T1-M1 cells. Representative bioluminescent images for lung metastasis in the mice are shown in Fig. 5B, and representative histological images of metastatic tumors in lung sections are presented 

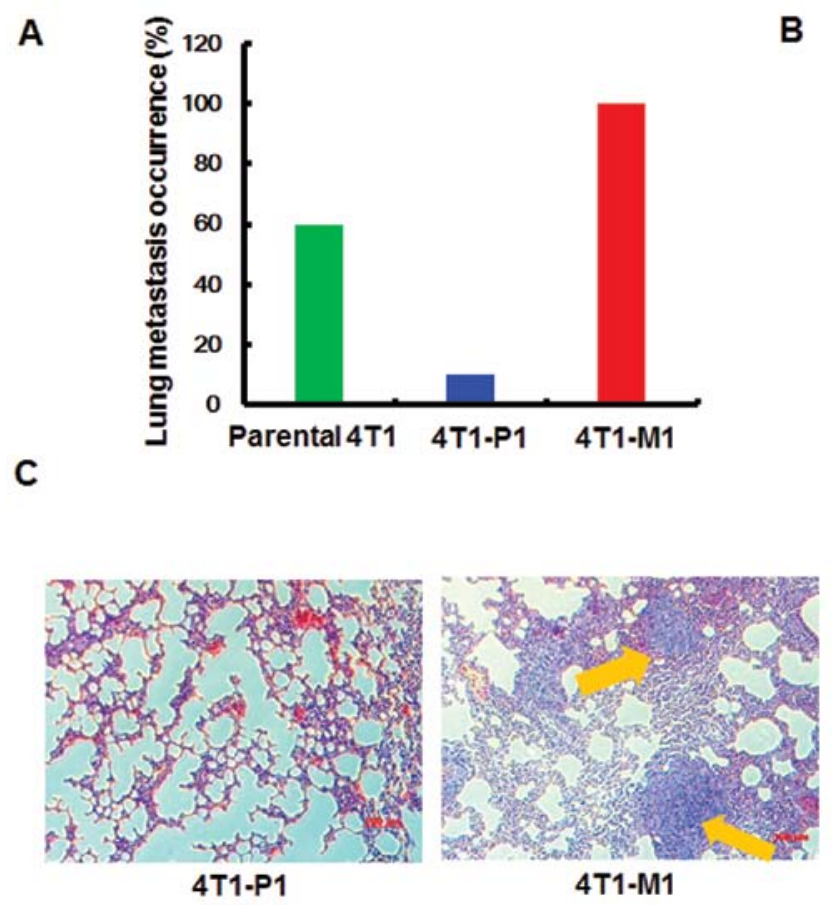

B

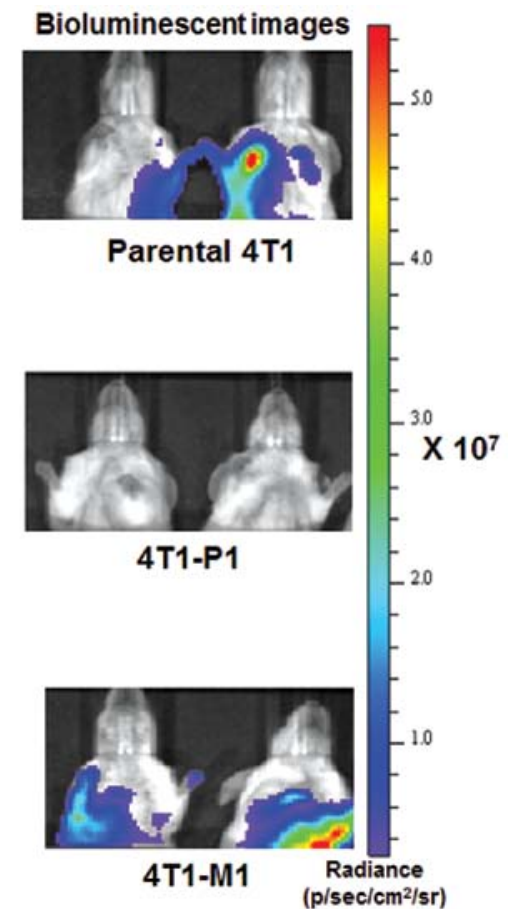

Figure 5. Lung metastasis in mice inoculated with 4T1-P1 or 4T1-M1 cells. (A) The overall occurrence of lung metastasis in mice upon inoculation of parental 4T1, 4T1-P1 or 4T1-M1 cells at the endpoint of the experiment. (B) Representative bioluminescent images for lungs from mice with implantation of parental 4T1, 4T1-P1 or 4T1-M1 cells. Positive bioluminescence is indicative of metastatic tumors with luciferase activity in lungs. (C) Representative histological examination of lung sections with H\&E staining. Original magnification, x100. Yellow arrows denote metastatic tumors in lung. H\&E, hematoxylin and eosin.

in Fig. 5C. These results clearly demonstrated that 4T1-M1 cells gained significantly enhanced propensity to metastasize to mouse lungs, and 4T1-P1 cells reversely lost the capability to metastasize. The parental $4 \mathrm{~T} 1$ cells presumably stayed in between with both properties of primary and metastatic tumors.

Enhanced epithelial-mesenchymal transition (EMT) for metastatic sublines. To identify the molecular mechanism underlying the elevated propensity of the metastatic sublines, we investigated the possible induction of EMT in these cells. EMT defines the conversion of epithelial cells into migratory and invasive mesenchymal cells. EMT is an inherent natural process during development for the generation of tissues and organs; however, it is an unintentional process in cancer progression, associated with enhanced capabilities in resistance to senescence upon chemotherapy and immunotherapy, stem cell properties, invasion and metastasis $(17,18)$. Snail1, the founding member of the Snail superfamily of zinc-finger transcription factors, plays a central role in the regulation of EMT (19). Snaill's EMT program is driven by a complex network involving several targets that are associated with reduction in cell adhesion, increase in migratory capacity, drug/immune resistance and gain of stem cell properties (20). As shown in Fig. 6A, a significant induction in Snaill was observed in the 4T1-M cells, compared to the 4T1-P cells, as shown by western blot analysis $(\mathrm{P}<0.05)$. This observation suggested that metastatic propensity was greatly enhanced in the 4T1-M cells, relative to the 4T1-P cells. In support of this observation, one of the Snaill targets, occludin was accordingly suppressed in the 4T1-M cells, compared to that in the 4T1-P cells (Fig. 6B, P<0.05). Consistent with our finding, a previous study revealed that the repression of occludin is due to the presence of Snail1 (21). Snail1 substantially inhibits the promoter activity of occludin by binding to the E-box located in its promoter region. Thus, Snaill serves as a transcriptional repressor of occludin (22). The reduction in occludin results in the loss of cell-to-cell adhesion that is necessary for the EMT process (22). Therefore, these results demonstrated that enhanced EMT could be a primary mechanism underlying the metastatic propensity of the $4 \mathrm{~T} 1-\mathrm{M}$ cells.

\section{Discussion}

To date, although the mechanisms underlying breast cancer metastasis remain elusive, metastasis-specific treatments would surely yield more effective therapeutic outcomes for breast cancer patients with metastatic tumors. Thus, the study of metastasis is critical. A pronounced impediment to this research is the lack of appropriate cell models. On the one hand, human breast cancer cells reveal poor metastasis and unstable metastatic features in immunocompromised mice. The current xenograft models therefore show limited ability to reflect the actual metastatic process (23). On the other hand, the interspecies use of human cells in mice may not be able to faithfully represent the complex biological process, particularly at the molecular level $(24,25)$. In contrast, murine breast cancer cells exhibit similar metastatic properties in mice to those as reported in breast cancer patients (26). In terms of mouse models, the 4T1 mouse model of breast cancer development and metastasis has been repeatedly used in a wide array of studies for more than 20 years. This cell line was originally established as a spontaneous breast cancer model in mice with the BALB/c genetic background (11). When implanted in syngeneic mice, the parental 4T1 cell line displays limited 
A

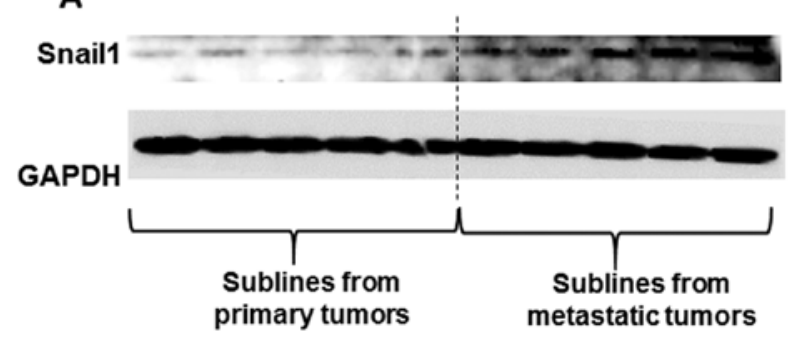

B

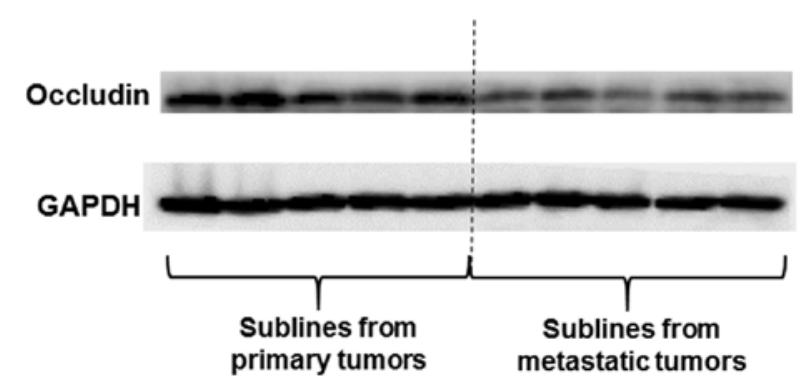

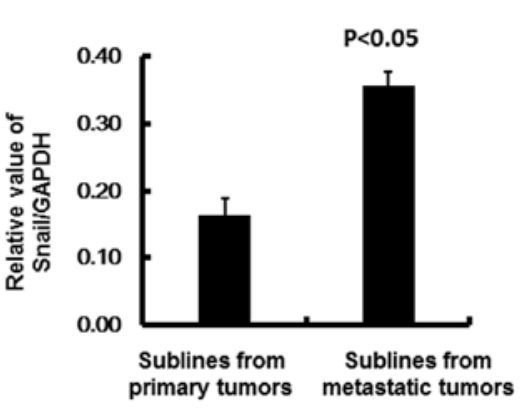

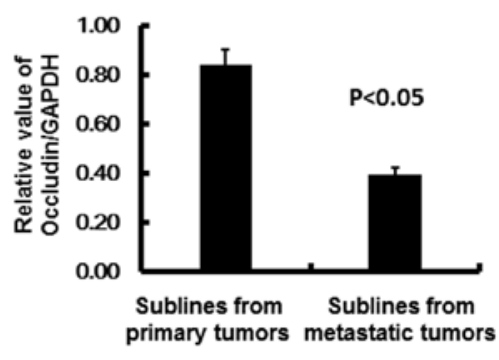

Figure 6. Determination of EMT markers in 4T1-P and 4T1-M cells by western blot analysis. (A) The concentration of Snail1 in sublines from primary and metastatic tumors. Quantified data are shown in the right panel $(n=5)$. (B) Occludin concentration in sublines from primary and metastatic tumors. The relative value was quantified for each group and shown in the right panel $(n=5)$. The intensity of the protein bands was calculated using ImageJ software. EMT, epithelial-mesenchymal transition.

and infrequent metastasis to local lymph nodes and distant organs $(7,11)$. To address this issue, in the present study, we established cell sublines of 4T1 cells with significantly metastatic propensity and specific tropisms to mouse lungs. To the best of our knowledge, this is a unique orthotopic model of mouse breast cancer metastasis to mouse lungs. These sublines would be beneficial to recognize the molecular events (such as gene expression signatures) responsible for mechanisms that drive the progression of metastasis.

As a main cause of cancer-related death, metastasis involves a serial of events: escape of cancer cells from a primary tumor, spread via the circulation and eventually colonization at distant sites, where some tumor cells acquire dormant or actively dividing phenotypes (27). EMT is considered as a driving force of breast cancer metastasis, as EMT changes cytoskeleton remodeling from apical-basolateral polarities indicative of epithelial cells to the generation of actin stress fibers characteristic of mesenchymal cells (18). As a result, EMT leads to the production of invasive and migratory tumor cells, with stem cell-like and chemoresistant properties $(18,28)$. In support of a contributive role of EMT in metastasis, clinical studies in patients with breast cancers indicate that enhanced concentration of mesenchymal markers in tumor cells is more predictive of poor prognosis than epithelial cytokeratin markers alone (29-33). EMT is a complex process involving a large number of genes, of which Snaill plays a crucial role in this event $(20,27)$. The primary contribution of Snaill in EMT is to regulate the expression of genes necessary for EMT including the repression of E-cadherin and occludin, leading to compromised cell adhesion and enforced migratory capacity (20). Increased cell motility and invasiveness in a primary tumor would certainly contribute to the dissemination of cancer cells to circulation and distant organs (34). E-cadherin is a transmembrane adhesion glycoprotein, which is exclusively expressed in epithelial cells (35). For example, E-cadherin is highly expressed in normal mammary ductal epithelial cells, whereas its expression is diminished in metastatic breast cancer cells (36). E-cadherin crucially governs calcium-dependent intercellular adhesion under normal settings (37). However, its expression is abnormally altered in cancer cells, and reduced expression oftentimes represents an invasive and metastatic phenotype (38-40). Previous studies indicate that aberrant expression of E-cadherin is associated with more invasive and metastatic breast cancers. Thus, reduced expression of E-cadherin has been reported to be associated with the development of distant metastases in patients with breast cancer, and E-cadherin has been recognized as a tumorsuppressor (38-40). Occludin is an integral transmembrane protein with four hydrophobic domains, and it is crucial for the integrity of tight junctions (41). Occludin is an important component at tight junctions in epithelial and endothelial cells (41), and loss of occludin causes loose cell-to-cell adhesion that would accelerate the EMT process during cancer progression (20). In the present study, Snail1 was identified to be greatly induced in the metastatic sublines, compared to cell lines from primary tumors. As the master regulator in EMT, its downstream target occludin was significantly repressed in metastatic sublines, compared to the control cell lines. These changes would greatly induce EMT and contribute to metastasis, supporting the enhanced propensity of metastasis for the metastatic sublines.

To summarize, we established a novel orthotopic model of breast cancer metastasis to mouse lungs. Metastatic sublines of 4T1 cells were characterized with substantial metastatic propensity to lungs, and these cells also displayed enhanced EMT features. This novel model allows detailed investigation of the molecular bases responsible for site-specific metastasis of breast cancer to the lungs. The present study also opens an emerging avenue for therapeutic studies in a more clinically relevant model. 


\section{Acknowledgements}

This study was supported by a grant under the National ' 973 ' Program (grant no. 2014CB932000), the Strategic Priority Research Program of the Chinese Academy of Sciences (grant no. XDB14000000), and grants from the National Natural Science Foundation of China (grant nos. 21377159, 21177151 and 21321004). We thank all the laboratory members for their great assistance with the experiments and reagents.

\section{References}

1. Siegel R, Naishadham D and Jemal A: Cancer statistics, 2013 CA Cancer J Clin 63: 11-30, 2013.

2. DeSantis C, Siegel R, Bandi P and Jemal A: Breast cancer statistics, 2011. CA Cancer J Clin 61: 409-418, 2011.

3. Lluch A, Cueva J, Ruiz-Borrego M, Ponce J and Pérez-Fidalgo JA Zoledronic acid in the treatment of metastatic breast cancer. Anticancer Drugs 25: 1-7, 2014.

4. Hayashi K, Aono M, Shintani K and Kazuki K: Bisphosphonate-related atypical femoral fracture with bone metastasis of breast cancer: Case report and review. Anticancer Res 34 $1245-1249,2014$

5. Minn AJ, Gupta GP, Padua D, Bos P, Nguyen DX, Nuyten D, Kreike B, Zhang Y, Wang Y, Ishwaran $\mathrm{H}$, et al: Lung metastasis genes couple breast tumor size and metastatic spread. Proc Natl Acad Sci USA 104: 6740-6745, 2007.

6. Minn AJ, Gupta GP, Siegel PM, Bos PD, Shu W, Giri DD, Viale A, Olshen AB, Gerald WL and Massagué J: Genes that mediate breast cancer metastasis to lung. Nature 436: 518-524, 2005.

7. Aslakson CJ and Miller FR: Selective events in the metastatic process defined by analysis of the sequential dissemination of subpopulations of a mouse mammary tumor. Cancer Res 52: $1399-1405,1992$

8. Tao K, Fang M, Alroy J and Sahagian GG: Imagable 4T1 model for the study of late stage breast cancer. BMC Cancer 8: 228 , 2008.

9. Pulaski BA and Ostrand-Rosenberg S: Reduction of established spontaneous mammary carcinoma metastases following immunotherapy with major histocompatibility complex class II and B7.1 cell-based tumor vaccines. Cancer Res 58: 1486-1493, 1998.

10. Eckhardt BL, Parker BS, van Laar RK, Restall CM, Natoli AL, Tavaria MD, Stanley KL, Sloan EK, Moseley JM and Anderson RL: Genomic analysis of a spontaneous model of breast cancer metastasis to bone reveals a role for the extracellular matrix. Mol Cancer Res 3: 1-13, 2005.

11. Miller FR, Miller BE and Heppner GH: Characterization of metastatic heterogeneity among subpopulations of a single mouse mammary tumor: Heterogeneity in phenotypic stability. Invasion Metastasis 3: 22-31, 1983.

12. Liu S, Goldstein RH, Scepansky EM and Rosenblatt M: Inhibition of rho-associated kinase signaling prevents breast cancer metastasis to human bone. Cancer Res 69: 8742-8751, 2009

13. Liu S, Li S and Du Y: Polychlorinated biphenyls (PCBs) enhance metastatic properties of breast cancer cells by activating Rho-associated kinase (ROCK). PLoS One 5: e11272, 2010.

14. Brocks C, Graefe H, Frenzel H, Pries R and Wollenberg B: Isolation of human myeloid dendritic cells from tumor tissue and peripheral blood. In Vivo 20: 239-242, 2006.

15. Padua D, Zhang XH, Wang Q, Nadal C, Gerald WL, Gomis RR and Massagué J: TGFbeta primes breast tumors for lung metastasis seeding through angiopoietin-like 4. Cell 133: 66-77, 2008.

16. Lelekakis M, Moseley JM, Martin TJ, Hards D, Williams E, Ho P, Lowen D, Javni J, Miller FR, Slavin J, et al: A novel orthotopic model of breast cancer metastasis to bone. Clin Exp Metastasis 17: 163-170, 1999.

17. Nieto MA: The ins and outs of the epithelial to mesenchymal transition in health and disease. Annu Rev Cell Dev Biol 27: 347-376, 2011.

18. De Craene B and Berx G: Regulatory networks defining EMT during cancer initiation and progression. Nat Rev Cancer 13: 97-110, 2013

19. Foubert E, De Craene B and Berx G: Key signalling nodes in mammary gland development and cancer. The Snaill-Twist1 conspiracy in malignant breast cancer progression. Breast Cancer Res 12: 206, 2010.
20. Kaufhold S and Bonavida B: Central role of Snaill in the regulation of EMT and resistance in cancer: A target for therapeutic intervention. J Exp Clin Cancer Res 33: 62, 2014.

21. Kajita M, McClinic KN and Wade PA: Aberrant expression of the transcription factors snail and slug alters the response to genotoxic stress. Mol Cell Biol 24: 7559-7566, 2004.

22. Ikenouchi J, Matsuda M, Furuse M and Tsukita S: Regulation of tight junctions during the epithelium-mesenchyme transition: Direct repression of the gene expression of claudins/occludin by Snail. J Cell Sci 116: 1959-1967, 2003.

23. Bibby MC: Orthotopic models of cancer for preclinical drug evaluation: Advantages and disadvantages. Eur J Cancer 40: 852-857, 2004

24. Parsons JT, Zetter B and Mohla S: Shifting paradigms in tumor metastasis: Challenges and opportunities. Cancer Biol Ther 1: $582-585,2002$.

25. Goldstein RH, Weinberg RA and Rosenblatt M: Of mice and (wo)men: Mouse models of breast cancer metastasis to bone. J Bone Miner Res 25: 431-436, 2010.

26. Vernon AE, Bakewell SJ and Chodosh LA: Deciphering the molecular basis of breast cancer metastasis with mouse models. Rev Endocr Metab Disord 8: 199-213, 2007.

27. Valastyan S and Weinberg RA: Tumor metastasis: Molecular insights and evolving paradigms. Cell 147: 275-292, 2011.

28. Ansieau S: EMT in breast cancer stem cell generation. Cancer Lett 338: 63-68, 2013.

29. Gradilone A, Raimondi C, Nicolazzo C, Petracca A, Gandini O, Vincenzi B, Naso G, Aglianò AM, Cortesi E and Gazzaniga P: Circulating tumour cells lacking cytokeratin in breast cancer: The importance of being mesenchymal. J Cell Mol Med 15: 1066-1070, 2011.

30. Roxanis I: Occurrence and significance of epithelial-mesenchymal transition in breast cancer. J Clin Pathol 66: 517-521, 2013.

31. Taube JH, Herschkowitz JI, Komurov K, Zhou AY, Gupta S, Yang J, Hartwell K, Onder TT, Gupta PB, Evans KW, et al: Core epithelial-to-mesenchymal transition interactome gene-expression signature is associated with claudin-low and metaplastic breast cancer subtypes. Proc Natl Acad Sci USA 107: 15449-15454, 2010.

32. Hennessy BT, Gonzalez-Angulo AM, Stemke-Hale K, Gilcrease MZ, Krishnamurthy S, Lee JS, Fridlyand J, Sahin A, Agarwal R, Joy C, et al: Characterization of a naturally occurring breast cancer subset enriched in epithelial-to-mesenchymal transition and stem cell characteristics. Cancer Res 69: 4116-4124, 2009.

33. Herschkowitz JI, Simin K, Weigman VJ, Mikaelian I, Usary J, $\mathrm{Hu}$ Z, Rasmussen KE, Jones LP, Assefnia S, Chandrasekharan S, et al: Identification of conserved gene expression features between murine mammary carcinoma models and human breast tumors. Genome Biol 8: R76, 2007.

34. Liu S: The ROCK signaling and breast cancer metastasis. Mol Biol Rep 38: 1363-1366, 2011.

35. Twiss F and de Rooij J: Cadherin mechanotransduction in tissue remodeling. Cell Mol Life Sci 70: 4101-4116, 2013.

36. Schneider MR and Kolligs FT: E-cadherin's role in development, tissue homeostasis and disease: Insights from mouse models: Tissue-specific inactivation of the adhesion protein E-cadherin in mice reveals its functions in health and disease. Bioessays 37: 294-304, 2015.

37. Nelson WJ, Dickinson DJ and Weis WI: Roles of cadherins and catenins in cell-cell adhesion and epithelial cell polarity. Prog Mol Biol Transl Sci 116: 3-23, 2013.

38. Jeanes A, Gottardi CJ and Yap AS: Cadherins and cancer: How does cadherin dysfunction promote tumor progression? Oncogene 27: 6920-6929, 2008.

39. Gheldof A and Berx G: Cadherins and epithelial-to-mesenchymal transition. Prog Mol Biol Transl Sci 116: 317-336, 2013.

40. Farahani E, Patra HK, Jangamreddy JR, Rashedi I, Kawalec M, Rao Pariti RK, Batakis P and Wiechec E: Cell adhesion molecules and their relation to (cancer) cell stemness. Carcinogenesis 35: 747-759, 2014.

41. Furuse M, Hirase T, Itoh M, Nagafuchi A, Yonemura S, Tsukita S and Tsukita S: Occludin: A novel integral membrane protein localizing at tight junctions. J Cell Biol 123: 1777-1788, 1993. 